\title{
A New Method to Measure the Distance Between Graduation Lines on Graduated Scales
}

\author{
William B. Penzes, Member, IEEE, Richard A. Allen, Member, IEEE, \\ Michael W. Cresswell, Senior Member, IEEE, Loren W. Linholm, Fellow, IEEE, \\ and E. Clayton Teague
}

\begin{abstract}
Line scales are used throughout industry for a variety of applications. The most common is the stage micrometer, a small, graduated glass scale for the calibration of optical instruments such as microscopes. However, stage micrometers are generally not calibrated, except for critical applications, due to the time and cost of optical calibration techniques. A method for calibrating line scales is presented which uses electrical test structure metrology. A description of the technique as well as examples of results from this technique are presented.
\end{abstract}

Index Terms - Electrical calibration, electrical test structure, laser interferometry, length, line scale, measurement uncertainty, optical calibration, scale graduations, stage micrometer, potentiometer, voltage-dividing.

\section{INTRODUCTION}

$\mathbf{L}$ INE SCALES in common use in industry include those with graduation pitch as small as $0.5 \mu \mathrm{m}$ to those as long as $1 \mathrm{~m}$. These scales are either linearly or, more rarely, logarithmically graduated. Smaller scales are made from glass or quartz substrates; longer scales are made from steel, invar, glass, quartz, or Zerodur [1] substrates. The best quality 1$\mathrm{mm}$ stage micrometers are produced using photolithographic etching of chromium on a glass or quartz substrate.

Presently, line scales are measured either by an optical comparator (for low accuracy) or by laser interferometry (for high accuracy). For a typical 1-mm stage micrometer, the spacings of the major subdivisions (100 $\mu \mathrm{m}$ nominal) are measured as well as the spacings of a single group of minor subdivisions (10 $\mu \mathrm{m}$ nominal). This calibration procedure is time consuming and, consequently, expensive. A simple, low-cost, electrical test structure-based calibration procedure for these line scales is described in this paper. Results of this calibration are compared with high-accuracy, laser-interferometry-based calculations.

Manuscript received April 18, 1996; revised September 3, 1999. This work was supported by the National Institute of Standards Technology. This paper was originally presented at the 1994 IEEE International Test and Measurement Conference, Hamatsu, Japan.

W. B. Penzes and E. C. Teague are with the Precision Engineering Division, National Institute of Standards and Technology, Gaithersburg, MD 20899 USA.

R. A. Allen, M. W. Cresswell, and L. W. Linholm are with the Semiconductor Electronics Division, National Institute of Standards and Technology, Gaithersburg, MD 20899 USA.

Publisher Item Identifier S 0018-9456(99)09422-X.

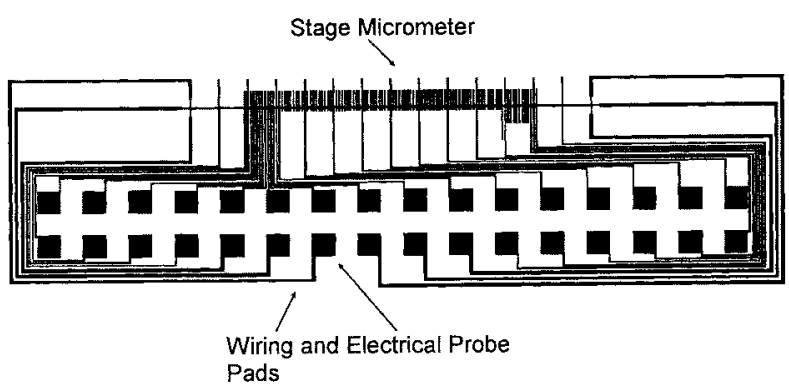

Fig. 1. The 1-mm NIST 20-stage micrometer.

\section{CALIBRATION PROCEDURE}

\section{A. Test Structure}

A 1-mm stage micrometer, designated NIST20 (Fig. 1), was designed for optical and electrical calibration. The electrical calibration technique is based on the modified voltage-dividing potentiometer [2] test structure, originally developed for VLSI lithography characterization. Fig. 2 highlights the key features of the NIST20 stage micrometer:

1) a horizontal bridge resistor;

2) voltage taps attached to selected graduations;

3) reference features (for analysis of the electrical measurements);

4) Van der Pauw crosses [3] to evaluate the uniformity of the conducting film.

The NIST20 stage micrometer was patterned at 10X, with e-beam lithography, in chrome on a quartz substrate. This $10 \mathrm{X}$ master was stepped across a second chrome-on-quartz substrate to produce the final $1 \mathrm{X}$ features.

\section{B. Laser Interferometric Calibration}

The NIST20 stage micrometer was measured by the NIST line scale interferometer (LSI) [4]. The NIST LSI consists of a scanning electro-optical line detector, a high precision one-axis motion system, and a high accuracy heterodyne interferometer for determining the displacement of the scale beneath the line detector. The wavelength of a stabilized helium-neon laser, corrected for temperature, relative humidity, and atmospheric pressure, is used as the length standard. The instrument is housed in an environmental chamber in which all environmental properties are carefully monitored. The frequency of the commercial laser used for the interferometer is calibrated 
against the NIST's iodine stabilized helium-neon laser as a frequency standard. The iodine stabilized helium-neon laser has a wavelength $\lambda=0.63299139822 \mu \mathrm{m}$ with an estimated relative standard uncertainty of $3.165 \times 10^{-11} \mu \mathrm{m}(1 \sigma)$ [5]. The wavelength of the light is computed from the vacuum wavelength of the iodine stabilized helium-neon laser and the index of refraction of the air along the light paths.

Each length value is the mean of eight measurements and the expanded standard uncertainty $(U)$ for each value is

$$
U=k u_{c}
$$

where the combined standard uncertainty $\left(u_{c}\right)$ is

$$
u_{c}=\sqrt{\left(u_{i}^{2}+u_{j}^{2}\right)}
$$

where $u_{i}$ is the standard uncertainty arising from random effects and $u_{j}$ is the standard uncertainty arising from systematic effects in the measurement process. The coverage factor $k=2$ was used which gives for each measured value a level of confidence of approximately 95\% [6]. Measurements were made from line center to line center by scanning a $0.08 \mathrm{~mm}$ long segment of each line midway between the graduation line tips and the horizontal base line.

All lengths are reported at a temperature of $20{ }^{\circ} \mathrm{C}\left(68^{\circ} \mathrm{F}\right)$. During the measurement the instrument and the scale temperature was held at $20 \pm 0.005{ }^{\circ} \mathrm{C}$, and the scale length was normalized to $20{ }^{\circ} \mathrm{C}$ using the coefficient of linear thermal expansion of the quartz substrate $\left(0.5 \times 10^{-6} /{ }^{\circ} \mathrm{C}\right)$. The measurements reported by the NIST LSI are all referenced to the $0.0 \mathrm{~mm}$ graduation line. Using this technique, this system is capable of routinely providing line spacing measurements with an expanded standard uncertainty of $10 \mathrm{~nm}$ for line spacings up to $100 \mathrm{~mm}$.

The time required to calibrate a scale, using the NIST LSI technique, is determined mainly by the setup time and the calibration time. Setup time needed for a scale in normal position includes scale inspection and preparation, scale supporting, scale alignment and focusing under the microscope, laser interferometer alignment, and instrument, and scale temperature stabilization. When the scale is placed in the reverse position the setup time is reduced to the time needed for scale alignment and focusing and instrument and scale temperature stabilization. The actual calibration for a stage micrometer, with 40 intervals, requires three hours, while the scale setup requires four and one half hours. It is clear that using the NIST LSI multipurpose, one-dimensional measuring instrument, which has a measuring range of $1 \mu$ to $1 \mathrm{~m}$, requires considerable setup time. But a small dedicated LSI used only for measuring stage micrometers would enable the setup time to be reduced by at least a factor of two. In the next section we describe an electrical calibration technique that considerably improves the total calibration time.

\section{Proposed Electrical Calibration Technique}

Electrical characterization is performed using a commercial parametric test system, with the following key features:

1) a low-noise switching matrix;

2) a high-linearity and accuracy (i.e., calibrated traceable to NIST) current source and microvolt meter.



Fig. 2. The active area of the NIST 20 stage micrometer showing key features.
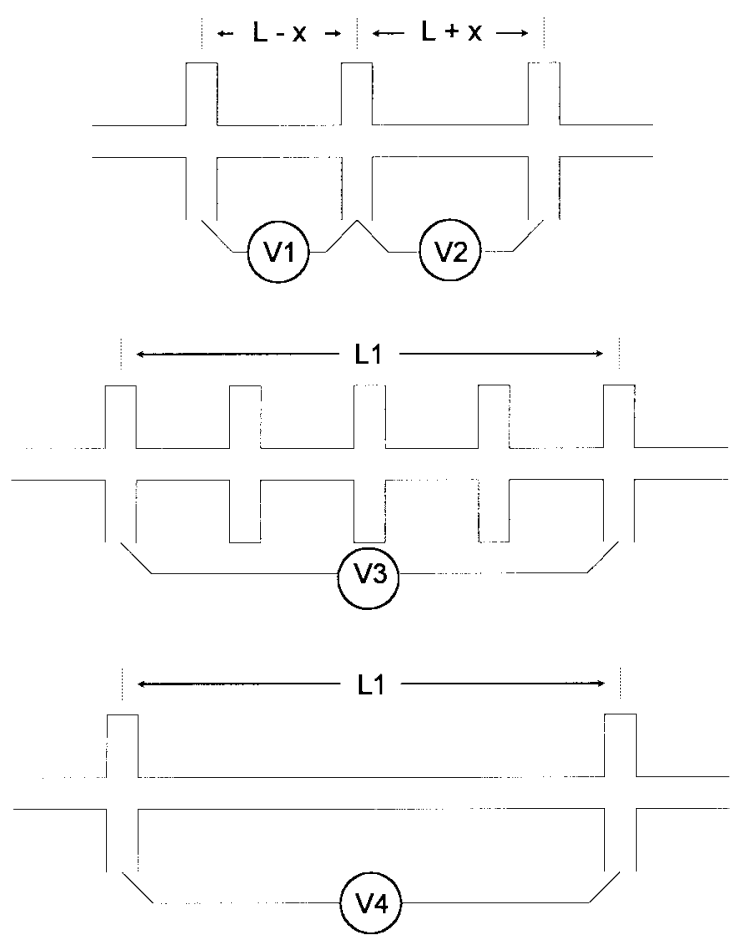

Fig. 3. Features of the modified voltage-dividing potentiometer.

Features of a generic potentiometer test structure are shown in Fig. 3. The displacement of the central tap from the midpoint of the end taps in Fig. 3(a) is given by

$$
x=\left(\frac{V_{1}-V_{2}}{V_{1}+V_{2}}\right)\left(\frac{L_{e}}{2}\right)
$$

where $L_{e}$ is the electrical length of the bridge. The electrical length differs conceptually from the physical length, i.e., that which an instrument such as the line scale interferometer would measure. The electrical length is defined as the physical length that an ideal feature of a measured series resistance and known and uniform width and sheet resistance would exhibit. It differs from the physical length when, for example, there is current shunting due to the discontinuities at the voltage taps, as occurs in this type of structure. For the features shown in Fig. 3(a), the electrical length is

$$
L_{e}=2 L-2 \delta L
$$

where $L$ is one-half of the physical length and $\delta L$ is the net effect of a single voltage tap on the length. Since $\delta L$ is dependent on the exact geometry of the intersection, it must be determined empirically. One method of determining $\delta L$ uses 
measurements of two additional features [Fig. 3(b) and (c)]. Here

$$
\delta L=L_{1}\left(\frac{V_{4}-V_{3}}{(n+1) V_{4}-V_{3}}\right)
$$

where $n$ is the number of "dummy taps" in Fig. 3(b).

Rather than the single, centered, interior tap of the generic structure, graduations within NIST20 are placed at $0.01 \mathrm{~mm}$ intervals along a 1-mm bridge in 10 major subdivisions of 10 segments each. A number of these graduations extend to probe pads for electrical measurement. Therefore an equation for $x$, more general than (3), was derived for arbitrary pairs of segments. The voltage drop along each segment, for a constant forced current, is proportional to the electrical length of the segment, i.e.,

$$
V_{l}=k\left(L_{l}-\sum_{l} \delta L x\right)
$$

and

$$
V_{r}=k\left(L_{r} \sum_{r} \delta L+x\right)
$$

where $k$ depends on the sheet resistance and bridge width and therefore is constant within a single bridge. The subscripts $l$ and $r$ refer to the left- and right-hand segments, respectively. The $L$ and $\delta L$ terms are the lengths of the segments and the total change in physical length due to the intervening taps, respectively. Taking the ratio of these two equations and solving for $x$ yields

$$
x=\frac{V_{r}\left(L_{l} \sum_{l} \delta L\right)-V_{l}\left(L_{r} \sum_{r} \delta L\right)}{V_{l}+V_{r}} .
$$

Unlike in the generic structure, interior segments of the graduated scale were used, in conjunction with the reference features, to determine $\delta L$.

The sources of uncertainty in the electrical measurements of the placement of the graduations were determined. The relative impacts of these factors are different for features of substantially different spacings. For the $0.01 \mathrm{~mm}$ spaced graduations, the uncertainty in the reference length [i.e., $L_{l}+$ $L_{r}$ in (8); assumed to be the design value] is dominant. Since the reference length uncertainty is constant within a single bridge, the effect will be less when finding the placements of the $0.10-\mathrm{mm}$ spaced graduations. For the $0.10-\mathrm{mm}$ spaced graduations, the primary source of uncertainty is due to instrumentation. The reason for this can be seen by considering of (8). Since $x$ is approximately zero, the terms in the numerator are approximately equal. Thus, for increased resistances the difference between the two terms as a percentage of the terms is smaller. This becomes the classic problem of accurately determining the small difference between two large measurements where the uncertainty of the instruments is a percentage of the measurement range and/or measured value. Additional sources of uncertainty in the electrical measurement are bridge nonuniformities and variations in $\delta L$.

During electrical testing, the temperature was approximately $23^{\circ} \mathrm{C}$. A typical time for electrical characterization of the
TABLE I

Calculated $\delta L$ 's and Standard Deviation $(\sigma)$

\begin{tabular}{l||c|c|c}
\hline \multicolumn{1}{c||}{ Tap Type } & Number of Segments & $\delta \mathrm{L}(\mu \mathrm{m})$ & $\sigma(\mu \mathrm{m})$ \\
\hline \hline Isolated & 2 & 0.973 & 0.051 \\
\hline End (long extensions) & 2 & 1.329 & 0.004 \\
\hline Transition & 2 & 1.356 & 0.006 \\
\hline Interior & 6 & 1.375 & 0.005 \\
\hline \hline
\end{tabular}

NIST20 stage micrometer is six minutes and the repeatability of electrical feature placement measurements, is approximately $0.8 \mathrm{~nm}(2 \sigma)$, where $\sigma$ is the average of repeated measurements.

\section{RESULTS}

\section{A. Length-Effect Parameter Calculation}

Analysis of the measurements showed that there was a large difference in the length effects of isolated voltage taps and interior voltage taps. Additionally, there were small differences between different groups of ten taps along the stage micrometer; in particular, between the end graduations (segments $\mathrm{A}$ and $\mathrm{J}$ in Fig. 2), transition graduations (segments $\mathrm{B}$ and I), and interior graduations (segments $\mathrm{C}-\mathrm{H}$ ). The different values for the $\delta L$ 's are shown in Table I. These differences were too large to be explained by either the graduation placement error (see the calibration results in the next section) or nonuniformities in the sheet resistance (which was uniform and measured to be $18.9 \Omega / \square$ ) and are likely due to lithographic proximity effects.

\section{B. Stage Micrometer Calibrations}

Results from optical (line scale interferometer) and electrical calibration of a NIST20 stage micrometer are shown in Table II. The optical measurements, which provide a baseline for comparison with the proposed electrical technique, show the graduations were generally placed close to the design values. Comparison of the differences in measured graduation placement between the two techniques provides an estimate of the uncertainty in the electrical measurements. The average differences are 0.009 and $0.028 \mu \mathrm{m}$ for the 0.01 - and the 0.1 -mm-spaced features, respectively.

\section{NeW Design}

It is likely that an enhanced design would reduce the measured differences between the optical and electrical measurements by reducing the variation in $\delta L$, due to lithographic proximity effects. Two modifications to the design shown in Fig. 2 are foreseen: first, extending the taps that extend a short distance below the bridge in Fig. 2 and second, placing one or more additional $0.1 \mu \mathrm{m}$ segments of ten graduations, which would not be used in the measurement, at each end of the stage micrometer. An additional enhancement would be to widen the bridge to lower the bridge resistance, thereby reducing the combined uncertainty in the measurements of the 0.10-mm-spaced segments. 
TABLE II

Graduation Spacings in the Nist20 Stage Micrometer

\begin{tabular}{c|c|c|c|c}
\hline \multirow{2}{*}{$\begin{array}{c}\text { Line Pitch } \\
\text { (nterval from } \\
0.000 \mu \mathrm{m})\end{array}$} & \multicolumn{2}{|c|}{ Line Scale Interferometer } & \multicolumn{2}{c}{ Voltage-Dividing Potentiometer } \\
\cline { 2 - 5 } & $\begin{array}{c}\text { Length } \\
\mu \mathrm{m})\end{array}$ & $\begin{array}{c}\text { Expanded } \\
\text { Uncertainty } \\
(\mu \mathrm{m})\end{array}$ & $\begin{array}{c}\text { Length } \\
(\mu \mathrm{m})\end{array}$ & $\begin{array}{c}\text { Difference } \\
(\mu \mathrm{m})\end{array}$ \\
\hline 10.000 & 10.005 & 0.004 & 10.004 & 0.001 \\
\hline 20.000 & 20.004 & 0.004 & 20.011 & -0.007 \\
\hline 30.000 & 30.005 & 0.008 & 30.037 & -0.032 \\
\hline 40.000 & 40.005 & 0.008 & 40.019 & -0.014 \\
\hline 50.000 & 50.003 & 0.008 & 50.006 & -0.003 \\
\hline 60.000 & 59.997 & 0.006 & 60.014 & -0.017 \\
\hline 70.000 & 69.997 & 0.006 & 70.008 & -0.011 \\
\hline 80.000 & 79.997 & 0.004 & 80.013 & -0.016 \\
\hline 90.000 & 89.996 & 0.006 & 90.010 & -0.014 \\
\hline \hline 100.000 & 99.997 & 0.006 & 99.971 & 0.026 \\
\hline 200.000 & 200.001 & 0.008 & 200.030 & -0.029 \\
\hline 300.000 & 300.003 & 0.008 & 300.002 & 0.001 \\
\hline 400.000 & 400.004 & 0.006 & 399.965 & 0.039 \\
\hline 500.000 & 499.999 & 0.008 & 499.947 & 0.052 \\
\hline 600.000 & 600.006 & 0.004 & 600.019 & -0.013 \\
\hline 700.000 & 700.003 & 0.008 & 699.978 & 0.025 \\
\hline 800.000 & 800.003 & 0.006 & 800.030 & -0.027 \\
\hline 900.000 & 900.007 & 0.004 & 899.971 & 0.036 \\
\hline 1000.000 & 1000.006 & 0.006 & $R e f e r e n c e$ Length & $($ Electrical $)$ \\
\hline \hline
\end{tabular}

\section{CONCLUSIONS}

The electrical metrology technique potentially provides quick and inexpensive calibrations of stage micrometers. While the accuracy provided by the line scale interferometer, a primary calibration system in a national standards laboratory, exceeds that provided by electrical test structure metrology, the accuracy provided by electrical test structure metrology likely exceeds the requirements of most applications and it has the benefit of using widely available commercial instrumentation. Thus, the electrical technique would be ideal for providing the general user access to inexpensive, calibrated artifacts.

The results of this experiment also suggest that an alternative process for production of graduated scales may lead to much more accurate feature placement. In particular, adoption of the VLSI production techniques (electron-beam lithography followed by photoreduction) may lead to extremely accurate graduated scales requiring minimal calibration for common applications.

\section{ACKNOWLEDGMENT}

The authors would like to thank W. Guthrie of the NIST Statistical Engineering Division for valuable technical discussions.

\section{REFERENCES}

[1] Zerodur is a trade name for a low thermal expansion coefficient glass manufactured by Schott Corporation.

[2] R. A. Allen, M. W. Cresswell, C. H. Ellenwood, and L. W. Linholm, "Voltage-dividing potentiometer enhancements for high-precision feature placement metrology," in Proc. IEEE 1992 Int. Conf. Microelectronic Test Structures, Mar. 1992, vol. 5, pp. 174-179.

[3] M. G. Buehler and W. R. Thurber, "A planar four-point test structure for measuring bulk resistivity," IEEE Trans. Electron Devices, vol. ED-23, pp. 968-974, Aug. 1976

[4] W. B. Penzes and J. S. Beers, "Evolution of automatic line scale measurement at the national institute of standards and technology," in IMECO Proc. Symp. Measurement Inspection Industry Computer Aided Laser Metrology, Sept. 1990, no. 28, pp. 41-58.

[5] T. J. Quinn, "Mise en practique of the definition of the meter (1992)," Metrologia 1993/94, vol. 30, pp. 523-541.

[6] B. J. Taylor and C. E. Kuyatt, "Guidelines for evaluating and expressing the uncertainty of NIST measurement results," NIST Tech. Note 1297, 1994 ed.

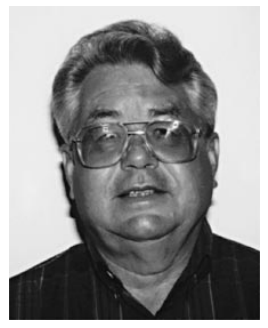

William B. Penzes (M'82) is an Electronics Engineer and Project Leader with the Precision Engineering Division, National Institute of Standards and Technology (NIST), Gaithersburg, MD, working in the field of high precision measurements of length standards. He is responsible for the NIST line scale interferometer and for calibration services for length scales. He is also involved in research and development of instrumentation for ultra precision dimensional measurements by means of laser interferometry, capacitance transducers, and their unusual applications. He has authored or coauthored 29 papers and owns four U.S. patents in fields related to his work.

Mr. Penzes received the U.S. Department of Commerce Bronze Medal and Gold Medal. He is a member of the American Society for Precision Engineering, and the Instrument Society of America.

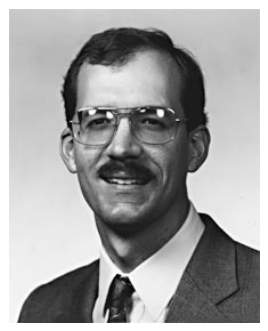

Richard A. Allen (M'85) received the B.S. and the M.S. degrees, both in physics, from Rensselaer Polytechnic Institute, Troy, NY.

He was with the Jet Propulsion Laboratory, Pasadena, CA, where he developed test structures for monitoring space radiation effects in the VLSI Technology Group and at College Park Software, Altadena, CA, where he worked on the development of LISP-based expert systems tools. Since June 1990, he has been with Integrated Circuits Technology Group of the National Institute of Standards and Technology (NIST), Gaithersburg, MD. His present interests include developing test structures for nanometer-level linewidth and overlay metrology.

Mr. Allen is a member of the American Physical Society.

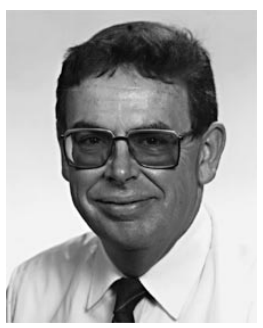

Michael W. Cresswell (M'66-SM'96) received the $\mathrm{Ph} . \mathrm{D}$. degree in physics from Pennsylvania State University, University Park, and the M.B.A. degree from the University of Pittsburgh, Pittsburgh, PA.

$\mathrm{He}$ is a Physicist in the Integrated Circuits Technology Group, National Institute of Standards and Technology (NIST), Gaithersburg, MD. He spent 19 years at Westinghouse working in bipolar powerdevices, flat panel displays, and IC-process development. At NIST he is developing reference material for nanometer-level metrology for submicrometer feature placement, linewidth, and overlay. He holds 20 patents in the filed of semiconductor-fabrication technology.

Dr. Cresswell is a member of the American Physical Society. 


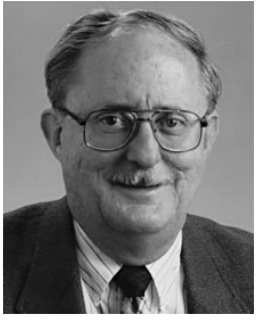

Loren W. Linholm (S67-M'68-SM'92-F'98) received the B.S. degree in electrical engineering from the University of California, Berkeley, CA, in 1968, and the M.S. degree in electrical engineering from the University of Maryland, College Park, in 1973.

He was with the Naval Missile Center, Point Mugu, CA, and the Department of Defense, Ft. Meade, MD. Since 1978, he has been with the Semiconductor Electronics Division, National Institute of Standards and Technology (NIST), Gaithersburg, MD. He is responsible for developing microelectronic test structures, test methods, a diagnostic procedure for characterizing and controlling integrated circuit manufacturing processes, evaluating tool performance, and improving device reliability.

Mr. Linholm is a member of the IEEE Electron Devices Society, Tau Beta $\mathrm{Pi}$, and Eta Kappa Nu.
E. Clayton Teague received the B.S., M.S., and Ph.D. degrees in physics.

$\mathrm{He}$ is Chief of the Automated Production Technology Division (APTD), Manufacturing Engineering Laboratory, National Institute of Standards and Technology (NIST), Gaitherburg, MD. Prior to being appointed to this position in January 1998, he served at NIST as a Group Leader and Researcher for 25 years making contributions in designing, constructing, and using precision instrumentation for ultra-high accuracy dimensional metrology of surfaces and micrometer to nanometer-scale discrete features. Precision instrumentation included scanning tunneling microscopes, displacement and phase-measuring interferometry, stylus instruments, flexure stages, light scattering apparatus, and ultra-high vacuum systems. Prior to his work at NIST, he had conducted research and development at Texas Instruments in semiconductor technology, primarily in vapor phase epitazial growth of silicon, germanium, and gallium arsenide. He has authored or coauthored 65 papers and presented 50 invited talks in the technical fields described

Dr. Teague has been awarded with the U.S. Department of Commerce Gold Medal, Silver Medal, and Allen V. Astin Measurement Science Award, the Kilby International Award by the Kilby Awards Foundation, and an IR-100 Award by the Journal Industrial Research and Development. He was one of the organizers of the American Society for Precision Engineering and has served twice as the Society's President. 